\title{
Management häufiger postoperativer Probleme und Komplikationen
}

\section{Teil 2}

\author{
C. J. Krones, C. D. Klink, A. Lambertz
}

Im 1. Teil des Beitrags (Krones et al. 2015) wurden vier Kasuistiken dargestellt, welche die häufig auftretenden Komplikationen Lungenembolie, Alkoholentzug, Nachblutungen und postoperatives Fieber behandeln. Im 2. Teil werden drei zusätzliche Fallbeispiele - Übelkeit und Erbrechen,
Anastomoseninsuffizienz, postoperative Schmerzen - und ihre Hintergründe sowie das diagnostische und therapeutische Vorgehen beschrieben, um weitere Prinzipien im Management postoperativer Komplikationen darzustellen.

\section{Übelkeit und Erbrechen: Magen-Darm-Atonie}

\section{Hintergrund und Klinik}

Postoperativ beträgt die Inzidenz von Übelkeit und Erbrechen 20-30\%, sodass diese Beschwerden zu den am häufigsten auftretenden Komplikationen zählen.

\section{- Postoperative Nausea and Vomiting (PONV)}

Frühpostoperativ ist die häufigste Ursache das PONV, das noch am OP-Tag auftritt. Dessen Entstehung ist im Detail weitgehend ungeklärt, lässt sich aber als eine vegetative Reaktion des Körpers auf die verabreichten Narkosemedikamente zusammenfassen. Obwohl PONV in aller Regel selbstlimitierend ist, können in seltenen Fällen schwerwiegende Komplikationen wie Atemwegsverlegungen mit Sauerstoffmangel auftreten.

Die Therapie besteht in der Gabe von Antiemetika und bei anamnestisch prädisponierten Patienten in der Modifikation des Anästhesieverfahrens als Prophylaxe. Risikofaktoren für das Auftreten von PONV zeigt Tab. 1.

\section{Abkürzungen}

$\begin{array}{ll}\text { BGA } & \text { Blutgasanalyse } \\ \text { BZ } & \text { Blutzucker } \\ \text { GI } & \text { gastrointestinal } \\ \text { GIT } & \text { Gastrointestinaltrakt } \\ \text { LA } & \text { Lokalanästhetikum } \\ \text { NRS } & \text { numerische Rating-Skala } \\ \text { NSAID } & \text { Non-steroidal anti-inflammatory Drugs } \\ & \text { (nichtsteroidale Antiphlogistika) } \\ \text { OPSI-Syndrom } & \text { Overwhelming post-Splenectomy Infection } \\ & \text { (Postsplenektomiesyndrom) } \\ \text { PCEA } & \text { patientenkontrollierte epidurale Analgesie } \\ \text { PDK } & \text { Periduralkatheter } \\ \text { PONV } & \text { postoperative Nausea and Vomiting } \\ & \text { (postoperative Übelkeit und Erbrechen) } \\ \text { RAK } & \text { Regionalanästhesiekatheter } \\ \text { RR } & \text { Blutdruck } \\ \text { VAS } & \text { visuelle Analogskala } \\ \text { VRS } & \text { verbale Rating-Skala } \\ \text { ZVK } & \text { zentraler Venenkatheter } \\ & \end{array}$




\section{Perioperative Medizin}

Tabelle 1

Risikofaktoren für das Auftreten von PONV (aus Rüsch et al. 2010).

\begin{tabular}{|c|c|}
\hline Gruppe & Risikofaktor \\
\hline patientenbedingt & $\begin{array}{l}\text { - weibliches Geschlecht } \\
\text { - Anamnese von PONV } \\
\text { - Reisekrankheit } \\
\text { - Nichtraucherstatus }\end{array}$ \\
\hline anästhesiebedingt & $\begin{array}{l}\text { - volatile Anästhetika } \\
\text { - Anästhesiedauer (Risikozunahme alle } 30 \text { Minuten } \\
\text { um ca. } 60 \% \text { ) } \\
\text { - Lachgas }\end{array}$ \\
\hline operationsabhängig & - Art der Operation \\
\hline allgemein & $\begin{array}{l}\text { - Opioidgabe postoperativ } \\
\text { - Opioidgabe intraoperativ }\end{array}$ \\
\hline
\end{tabular}

\section{Magen-Darm-Atonie}

Nach größeren operativen Eingriffen gehört es zusätzlich zum „Normalverlauf“, dass die peristaltische Bewegung des Magen-Darm-Traktes für 12 bis maximal 72 Stunden herabgesetzt oder sogar praktisch aufgehoben ist. Diese prolongierte postoperative Störung der koordinierten gastrointestinalen Funktion ohne mechanisches Hindernis wird als Magen-Darm-Atonie bezeichnet. Sie ist fast immer reversibel. Nur selten geht die Störung der Transportfunktion in einen paralytischen Ileus über, und wirklich nur ganz selten wird daraus ein Obstruktionsileus.

Vermittelt wird die postoperative Atonie durch neurogene und inflammatorische Reaktionen auf den abdominalchirurgischen Eingriff. Vereinfachend kann man auch annehmen, dass der Stress eines operativen Eingriffs über die Schmerzafferenzen den Sympathikus stimuliert, welcher wiederum die Stilllegung des Magen-Darm-Traktes induziert (Abb. 1). Der später einsetzende, zweite Mechanismus, welcher zur Darmlähmung beiträgt, entspricht

Fallbeispiel 1

Ein 54-jähriger Patient klagt am 4. postoperativen Tag nach großflächiger Reparation einer Narbenhernie mittels retromuskulärer Netzplastik (Sublay-Technik) und ausgedehnter Adhäsiolyse schon nachmittags über hartnäckige Übelkeit. In der frühen Nacht erbricht er dann mehrfach schwallartig. Beim Eintreffen des Dienstarztes ist das Abdomen gebläht und ubiquitär druckempfindlich. Die Wunde ist reizlos. Es liegen noch 2 subfasziale Drainagen, die allerdings nur noch wenig serös-blutiges Sekret fördern. Die Vitalparameter sind unauffällig.

Der bisherige postoperative Verlauf war eigentlich unkompliziert, aber bereits am Morgen hatte der Mann nur sehr sparsam gefrühstückt. Auskultatorisch sind kaum Darmgeräusche auszumachen. Der Patient hatte bislang noch keine Stuhlentleerung. Der einliegende Periduralkatheter (PDK) läuft, der Patient gibt sich mit der Schmerztherapie jedoch nicht ausreichend zufrieden.

Bei der Durchsicht der Akte fallen zusätzlich eine fest angeordnete Begleitmedikation mit Metamizol und regelmäßige Gaben einer Bedarfsmedikation mit Morphin auf.
Der Dienstarzt stellt die Verdachtsdiagnose einer postoperativen Atonie nach Adhäsiolyse und Narbenhernienreparation. Sonografisch bestätigt sich die Diagnose schnell. Der Magen ist prall gefüllt, es findet sich kaum Darmperistaltik. Der Dünndarm ist ubiquitär dilatiert und pendelt nicht. Hungerdarm wird nicht dargestellt.

Nach Einlage einer Magensonde entleeren sich ca. 1,5 I Mageninhalt - der Patient fühlt sich anschließend deutlich besser. Aber die Bauchdecke schmerzt immer noch. Schmerzen und Atonie trotz einliegendem PDK erscheinen dem Diensthabenden nicht logisch. Deshalb wird der Schmerzdienst informiert und zur Überprüfung des PDK gebeten.

Zur Überbrückung wird eine Infusion mit Metamizol angeordnet. Das lindert die Beschwerden leidlich. Nachdem die Anästhesistin erscheint, zeigt sich schnell: Der PDK funktioniert nicht ausreichend. Der Patient leide vielleicht deshalb an Atonie und Schmerzen, so die Kollegin vom Schmerzdienst.

Die Anästhesistin spritzt den Katheter auf und erhöht auch die Flussrate. Sie verlässt die
Station mit der Empfehlung, ggf. 40 Tropfen Metamizol dazu zu geben. Der diensthabende Chirurg verzichtet darauf, die junge Kollegin über den Konflikt von Resorption, Atonie und Magensonde aufzuklären. Die Begleitmedikation bleibt intravenös. Die Nachtwache legt zusätzlich „feuchte Wärme“ auf, das lindert die Symptome. Der Patient führt schließlich noch in der Nacht erstmals etwas ab.

Bei der Morgenrunde des Diensthabenden sitzt er wieder auf dem Toilettenstuhl die Stuhlportion ist jetzt angemessen. Der Diensthabende lässt die Magensonde, die nur galliges Sekret fördert, abklemmen und erlaubt das schluckweise Trinken, was vor allem das Trockenheitsgefühl im Mund lindert. Am Nachmittag wird die Sonde entfernt und ein vorsichtiger Kostaufbau gestartet.

Der Patient erholt sich im weiteren Verlauf unkompliziert und verlässt das Haus am 10. postoperativen Tag. 


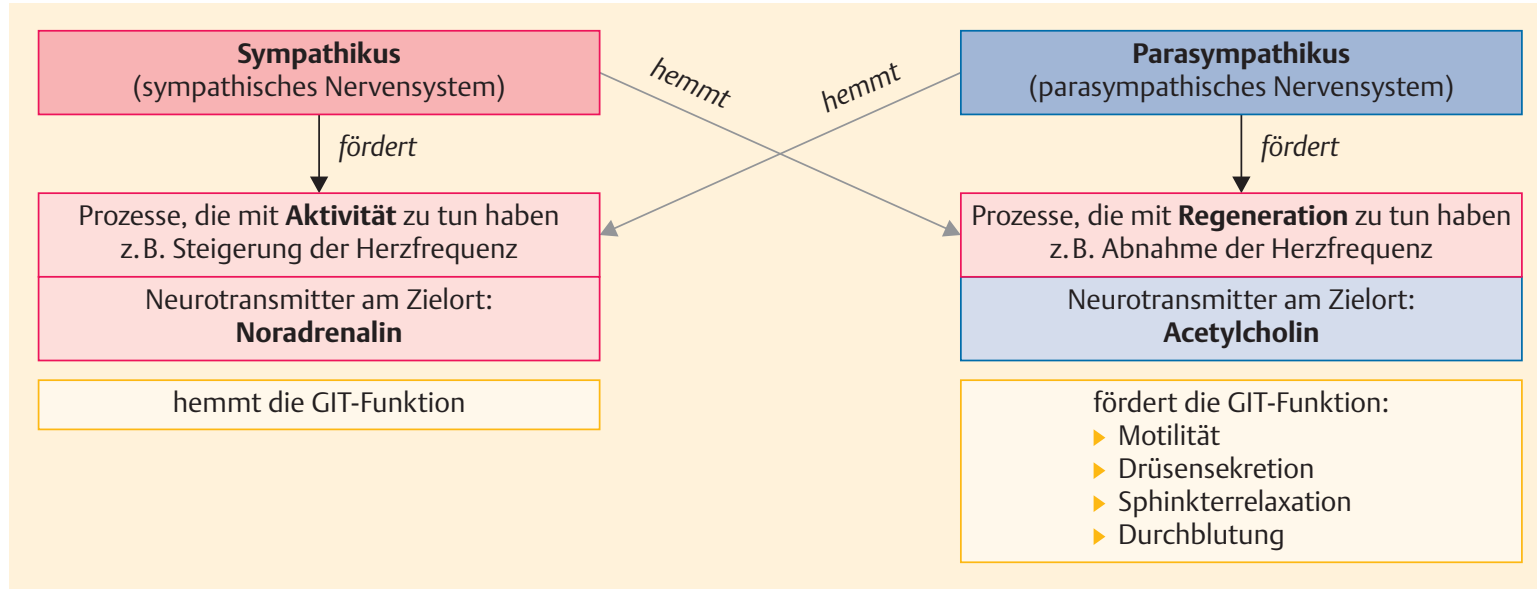

Abb. 1 - Entstehung der Atonie im Magen-Darm-Trakt.

einer immunologischen Reaktion. Beide Effekte interagieren bilateral.

Klinisch zeigt sich die Atonie durch ein aufgetriebenes Abdomen und dauerhaft fehlende Darmperistaltik. Der Übergang zum paralytischen Ileus manifestiert sich dann über eine Magenretention, die sich spät durch schwallartiges Erbrechen entleert. Ein früheres Warnsymptom ist übrigens ein persistierender Singultus. Hier reizt die Überfüllung des Magens das Zwerchfell.

Eine gut funktionierende patientenkontrollierte epidurale Analgesie (PCEA) über einen einliegenden Periduralkatheter (PDK) kann nach vielen abdominalchirurgischen Eingriffen nicht nur die Analgesie des Patienten wesentlich erleichtern, sondern auch das Risiko einer Atonie reduzieren. Das epidural applizierte Lokalanästhetikum (z.B. Ropivacain) blockiert die Schmerzafferenzen und damit im 2. Schritt die Aktivierung des Sympathikus. Der Parasympathikus wird in seiner Wirkung nicht antagonisiert und kann die Peristaltik fördern (Abb. 1).

Die PDK-Analgesie vermindert effizient die postoperative Atonie.

Die Wirkbreite des PDK ist allerdings segmental begrenzt. Um eine Sympathikolyse im Magen-Darm-Bereich zu erreichen, muss der PDK thorakal einliegen. Sehr breite Wundflächen oder Operationen im kleinen Becken benötigen aus gleichem Grund zur Analgesie immer eine ergänzende Begleitmedikation. Hier sollte man vorzugsweise auf periphere Analgetika zurückgreifen, die keine darmlähmende Nebenwirkung haben, wie z.B. Metamizol, Paracetamol oder NSAID-Präparate.
Kontraindikationen zur Anlage eines PDK sind:

- Gerinnungsstörungen,

- Rötungen an der Einstichstelle,

- Fieber und

- mangelnde Compliance bzw. Ablehnung durch den Patienten.

Die Peristaltik im Magen-Darm-Trakt wird außerdem durch eine inflammatorische Reaktion der Darmwand behindert. Vereinfacht kann allein der mechanische Reiz im Darmrohr Makrophagen, dendritische Zellen und Mastzellen so stimulieren, dass sie in der Darmwand eine inflammatorische Redaktion hervorrufen, die von molekularen Mediatoren wie Interleukinen, Stickoxid oder Cyclooxygenasen getragen wird. Auch wenn das komplexe Zusammenspiel der immunologischen Abläufe noch nicht im Detail aufgelöst ist, wurde einige Faktoren, die zur Entwicklung des postoperativen Ileus beitragen, bereits identifiziert. Die zielgerichtete Immunmodulation stellt eine der erfolgversprechenden Forschungsoptionen in der Behandlung der postoperativen Atonie dar.

\section{- Weitere Ursachen für postoperative Übelkeit und Erbrechen}

Weitere Ursachen sind Blutzuckerentgleisungen, Hypotonie sowie Medikamente.

Cave. Gerade die Nebenwirkung verabreichter Medikamente darf dabei nicht unterschätzt werden. 


\section{Übersicht}

\section{Häufige Ursachen von postoperativer Übelkeit und Erbrechen}

- Postoperative Nausea and Vomiting (PONV)

- Magen-Darm-Atonie

- Blutzuckerentgleisung

- Hypotonie

- medikamenteninduziert

\section{Diagnostisches Vorgehen}

Die Diagnose der beiden häufigsten Ursachen von postoperativer Übelkeit und Erbrechen - nämlich PONV und Magen-Darm-Atonie - ergibt sich meist aus Anamnese, klinischer Untersuchung und Sonografiebefund.

Die regelmäßige Auskultation, Palpation und Perkussion des Abdomens liefern wertvolle Hinweise zur Früherkennung. Dabei ist es übrigens völlig unerheblich, ob man zuerst palpiert oder auskultiert. Wenn die Peristaltik durch die Palpation angeregt wird, ist das ein gutes Zeichen und maskiert nichts.

Weitere Warnsignale sind Inappetenz, häufiges Aufstoßen und Schluckauf („da staut sich was!“).

Die beste Bildgebung stellt die Sonografie dar. Hier sieht man entweder eine ubiquitäre Darmdilatation mit träger oder fehlender Peristaltik oder wegen Luftüberlagerung aufgrund von Überblähung schlicht gar nichts.

Eine Röntgenleeraufnahme des Abdomens bietet dagegen kaum helfende Informationen. Ziemlich sicher wird ein vermehrter Luftgehalt im Magen-Darm-Trakt dargestellt werden. Die Lokalisation der Luft lässt keine Rück-

\section{Checkliste}

\begin{tabular}{|l|}
\hline Warnsignale für Atonie \\
\hline geblähtes Abdomen \\
fehlende Peristaltik \\
Inappetenz \\
Aufststoßen \\
Singultus \\
\hline
\end{tabular}

schlüsse zu. Frühpostoperativ wird auch noch freie Luft dabei sein - das verwirrt nur. Außerdem können sich Spiegel entwickeln, die dann ebenfalls in die falsche Richtung eines mechanischen Ileus weisen. Das dynamische Krankheitsbild lässt sich in einer Momentaufnahme nicht gut abbilden. Diese Strahlenbelastung kann man dem Patienten ersparen.

Die dynamische Sonografie ist dem Röntgen hoch überlegen. Nur hier lässt sich die Atonie auch vom mechanischen Ileus abgrenzen. Pendelperistaltik und vor allem Hungerdarm sind wichtige Hinweise auf eine obstruktive Passagebehinderung, die eine operative Therapie benötigt.

- Die Sonografie ist dem Röntgen in der Diagnostik der Darmpassagestörung deutlich überlegen!

\section{Therapeutisches Vorgehen}

Die Behandlung der Atonie und ihrer Symptome ist anspruchsvoll und vielgestaltig. Der vorliegende Fall ist einfach: Hier funktioniert die PCEA nicht ausreichend. Mit der Korrektur dieses Defizits erholt sich der Patient auch zügig. Der entscheidende Hinweis war der persistierende Schmerz. Die Therapie der Atonie ist ansonsten aber nicht so trivial. Sie gliedert sich in eine Symptomlinderung, abführende Maßnahmen und die Metaphylaxe eines Rezidivs.

\section{- Symptomlinderung der Atonie und Übelkeit}

An erster Stelle steht auch hier die Analgesie. Der distendierte Bauch schmerzt zwangsläufig.

Bevorzugt werden Analgetika, die möglichst keine Paralyse unterstützen, also eher keine Morphine. Allerdings müssen sich Präparat und Dosis an der Schmerzstärke ausrichten, d.h. sehr einfach: Starke Schmerzen fordern starke Analgetika. Die beste Steuerung gelingt dann doch mit Morphinen. Die klassische Nebenwirkung bleibt die Passageverzögerung. Sie variiert je nach Wirkstoff und weist dabei erhebliche Unterschiede auf, die man, ebenso wie das analgetische Äquivalent der Morphinpräparate, kennen muss.

Alle Morphine führen über ihre anticholinerge Nebenwirkung tendenziell zur Passageverzögerung!

Die zweite, sehr wichtige Maßnahme ist die Linderung der quälenden Übelkeit. Hier stehen unterschiedliche Präparate zur Verfügung, die im Einzelfall auch miteinander kombiniert werden müssen. Bei allen Präpara- 
ten muss man die Nebenwirkungen beachten, die zum Teil kontraproduktiv sind (Tab. 2).

Bei der Applikation muss natürlich die parenterale Form vorgezogen werden, da die Atonie auch die Resorption oral verabreichter Medikamente stark beeinträchtigt.

\section{- Abführende Maßnahmen}

Die Möglichkeiten der abführenden Maßnahmen gliedern sich in mechanische und medikamentöse Interventionen.

\section{Mechanische Anregung}

Mechanisch kann man den Darm auf unterschiedliche Arten reizen:

- Dehnung des Analsphinkters,

- Suppositorium, Klysma, Einlauf,

- Laxans oral,

- feuchte Wärme auf das Abdomen,

- intestinale Füllung.

Allen Maßnahmen ist in der Atonie zu eigen, dass sie die eigentliche Ursache der fehlenden Spontanaktivität des Darms nicht beheben. Das bedeutet: Sie lindern die aktuelle Notsituation, aber können allein nicht zum dauerhaften Erfolg führen.

\section{Medikamentöse Anregung}

Eine medikamentöse Anregung der Darmperistaltik ist noch schwieriger. Alle auf dem Markt befindlichen Präparate besitzen nur eine eingeschränkte Wirksamkeit (Tab. 3). Zum Teil wurde die Zulassung wegen des Nebenwirkungsprofils mittlerweile stark eingeschränkt. Die Autoren halten sie im Zweifel alle für verzichtbar.

\section{Magensonde}

Bei einer ausgeprägten Magenretention ist die Einlage einer Magensonde zu bedenken. Damit soll das quälende Erbrechen gestillt und eine stille Aspiration verhindert werden. Allerdings muss man beachten, dass der Patient an einer Magensonde entlang still aspirieren kann. Zudem sind die Sonden sehr unkomfortabel. Die Indikation muss also streng geprüft werden.

\section{- Metaphylaxe der Atonie}

Die beiden einzigen Maßnahmen zur Vermeidung einer Atonie, die neben der Analgesie eine nachgewiesene Wirksamkeit haben, sind

- die enterale Füllung und

- die Vermeidung der Überinfusion.

\section{Tabelle 2}

Übersicht Antiemetika.

\begin{tabular}{|c|c|c|}
\hline Substanzgruppe & Wirkstoff (Beispiel) & $\begin{array}{l}\text { Nebenwirkungen, } \\
\text { Anmerkungen }\end{array}$ \\
\hline \multirow[t]{3}{*}{ Kortikosteroide } & Dexamethason & BZ-Erhöhung \\
\hline & & RR-Erhöhung \\
\hline & & Mechanismus unklar \\
\hline \multirow[t]{2}{*}{ Serotoninantagonisten } & Odansetron & Obstipation \\
\hline & & Kopfschmerzen \\
\hline \multirow[t]{3}{*}{ Dopaminantagonisten } & Droperidol & Sedierung \\
\hline & & extrapyramidale Störung \\
\hline & & nicht bei Kindern einsetzen \\
\hline Histaminantagonisten & Dimenhydrinat & Sedierung \\
\hline \multirow[t]{2}{*}{ Anticholinergika } & Scopolamin & Schwindel \\
\hline & & Mundtrockenheit \\
\hline \multirow[t]{2}{*}{ Neurokininantagonisten } & Aprepitant & Kopfschmerzen \\
\hline & & Obstipation \\
\hline
\end{tabular}

Tabelle 3

Medikamentöse Anregung der Darmperistaltik.

\begin{tabular}{|l|l|}
\hline Wirkstoff & Wirkprinzip \\
\hline Bisacodyl & Laxans \\
\hline Na-Picosulfat & \\
\hline Macrogol & Motilinrezeptorantagonist \\
\hline Mg-Salz & Prokinetikum \\
\hline Erythromycin & \\
\hline Metoclopramid & \\
\hline Domperidon & Cholinesterasehemmer \\
\hline Neostigmin & \\
\hline
\end{tabular}


Grundlage ist hier eine Art Frank-Starling-Mechanismus: Um kontrahieren zu können, benötigt der Darm wie auch das Herz oder andere Hohlorgane eine Vorfüllung, d.h. ohne Nahrung keine Peristaltik. Die Vordehnung darf aber nicht so weit gehen, dass die Muskelfasern nicht mehr kontrahieren können. Erneut wie am Herzen bei der übermäßigen Dilatation kann sich der Darm in der Überdehnung nicht mehr bewegen. Diese Situation kennzeichnet den späten Ileus, lässt sich iatrogen aber auch durch eine Ödematisierung der Darmwand, z. B. in der überhöhten Plusbilanzierung, provozieren. Die Patienten lagern das überschüssige Wasser nicht nur in Lunge, Pleura, Aszites und Anasarka ab, sondern auch in der Darmwand. Die Situation lässt sich im Rahmen von Notfalllaparotomien schwerstkranker Patienten oftmals intraoperativ beobachten. Infusionen verschieben über den Natriumgehalt aber auch das Elektrolytgleichgewicht in der Darmwand. Analog zur Arrhythmie am Herzen führt das zu Störungen der Na/K- bzw. Ca-Pumpen, die an der Zellmembran das zur Kontraktion notwendige Aktionspotenzial steuern.

Beide Phänomene sprechen für das Behandlungsprinzip des Fast-Track, das u.a. einen frühen Kostaufbau mit zeitnaher Reduktion der parenteralen Ernährung beinhaltet.

- Früher Kostaufbau und begrenzte Infusionsmengen schützen vor der Atonie!

\section{Weitere Ursachen}

Seltenere Ursachen der postoperativen Übelkeit sollten immer differenzialdiagnostisch einbezogen werden, lassen sich aber zumeist leicht, z.B. durch eine Blutzuckermessung, ausschließen.

Die emetische Nebenwirkung von Medikamenten wird oft unterschätzt. Im Zweifel ist jedes Präparat geeignet, Übelkeit zu erzeugen. In der Viszeralchirurgie sollte man in diesem Kontext die Indikation von Antibiotika besonders kritisch prüfen.

\section{Insuffizienz nach Pankreaschirur- gie: lebensbedrohliche Blutung}

\section{Hintergrund und Klinik}

Obwohl in den letzten Jahrzehnten in der Pankreaschirurgie konsequente Fortschritte erzielt wurden, zählen diese Eingriffe weiterhin zu den komplikationsträchtigsten viszeralchirurgischen Operationen. Auch in spezialisierten Zentren mit hohen Eingriffszahlen kann die Mor-

\section{Überblick}

\section{Die wichtigsten Komplikationen \\ der Pankreaschirurgie}

- Fistel

- Blutung

- Magenentleerungsstörung

biditätsrate je nach Literatur zwischen $20-50 \%$ betragen Dabei hängt die Spannbreite der Daten an der Definition der Komplikation, sodass zur Verbesserung der Vergleichbarkeit die wichtigsten Komplikationen (s. Infobox „Überblick“) mittlerweile im internationalen Konsens festgelegt wurden. Allein die Mortalität ließ sich drastisch senken und liegt in High-Volume-Kliniken mittlerweile bei unter $5 \%$. Vor allem Fortschritte in der perioperativen Betreuung und dem Komplikationsmanagement waren hier entscheidend.

Die Komplikationsraten in der Pankreaschirurgie werden je nach Studie stark von der Komplikationsdefinition beeinflusst.

Außerhalb von kardiovaskulären Notfällen stellt eine Insuffizienz der Pankreasanastomose mit konsekutiver Fistel den vital gefährlichsten Teil des Eingriffs dar. Grundlagen sind vereinfachend:

- eine ungünstige Organstruktur des Pankreas und

- die Aggressivität des Pankreassekrets.

Die verschiedenen in der Literatur beschriebenen Risikofaktoren für eine postoperative Pankreasfistel sind in der Box zusammengefasst.

\section{Risikofaktoren}

Risikofaktoren für eine postoperative Pankreasfistel

- schmaler Pankreasgang $<5 \mathrm{~mm}$

- weiches Pankreasgewebe

- höherer Blutverlust

- Linksresektion

- BMI und Stärke der perirenalen Fettschicht

- männliches Geschlecht

- niedriges Serumalbumin, hohes Bilirubin

- Tumorstadium 
Der Dienstarzt wird am frühen Abend vom Spätdienst zu einem 69-jährigen Mann auf die Normalstation gebeten. Die Schwester bleibt am Telefon etwas vage: Der Patient befinde sich am 6 . Tag nach pyloruserhaltender Pankreasresektion eigentlich nicht in wirklich schlechter Verfassung. Er gefalle ihr klinisch aber einfach nicht, und der gestrige Tag sei besser gewesen. Der Chirurg bittet zunächst, die üblichen Vitalparameter - Puls, Blutdruck, Sättigung - zu erheben.

Vor Ort bleibt die Situation zunächst weiter unklar. Der Patient ist schwer einzuschätzen. Das Abdomen imponiert gebläht und fast etwas prall. Die Bauchdecken sind druckschmerzhaft, aber sicher nicht peritonitisch. Auskultatorisch ist es eher still, aber der Mann hat am Vortag etwas abgeführt und klagt nicht über Völlegefühl oder Aufstoßen. Die Wunde ist ganz reizlos, die Drainage fördert seröses Sekret. Nichts wirklich Fassbares.

Die Situation klärt sich auch nicht, als die Pflegekraft die Vitalwerte durchgibt. Der Blutdruck misst 100/60 mmHg, der Puls liegt bei 100 Schlägen pro Minute und die Sauerstoffsättigung bei $94 \%$. Die Zunge wirkt etwas trocken, dazu leichte Anasarka, nicht ungewöhnlich nach diesem Eingriff. Vielleicht etwas intravasales Volumen.
Der Diensthabende entscheidet sich für zusätzliche $500 \mathrm{ml}$ Flüssigkeit, nimmt aber vorher noch eine Blutprobe zur Laborkontrolle ab. Der Hämoglobingehalt ist von 9,3 mg\% am Morgen auf 7,0 mg\% gefallen. Parallel geht es dem Mann fast zusehends schlechter.

Jetzt muss gehandelt werden. Der Chirurg ordert das Sonografiegerät und lässt zeitgleich 4 Erythrozytenkonzentrate einkreuzen. Bei deutlicher Luftüberlagerung gelingt im Ultraschall jedoch auch keine wegweisende Aussage. Das Restpankreas selbst ist nicht einsehbar. Im Unterbauch schwimmt etwas freie Flüssigkeit, der Rest des Abdomens wirkt unübersichtlich, eher dicht gepackt, und hat Binnenechos. Doch zu viel Stuhlfüllung?

Der Patient wird schweißig, die Schwester drängt, der Chirurg zaudert. Mit dem Plan, eine CT-Untersuchung des Abdomens zu veranlassen, informiert der Dienstarzt telefonisch seinen Hintergrund. Der erfahrene Oberarzt stellt nach kurzem Bericht die Verdachtsdiagnose auf eine intraabdominelle Blutung: Also Notfall-OP statt Notfall-CT!

Ohne noch weitere Zeit zu verlieren geht es jetzt in den OP-Trakt. Auf dem Weg in den Saal trübt der Patient etwas ein - schnelle Blutdruckmessung während der Aufzugfahrt: $70 / 40 \mathrm{mmHg}$ ! Bis zur Schleuse wird gerannt.
Nach der Narkoseeinleitung ist der Patient bereits deutlich katecholaminpflichtig. Die aktuelle BGA bietet jetzt einen Hb-Wert von 4,9 mg\%. Der Bedarf an Kreislaufunterstützung steigt mit der Relaparotomie nochmals an.

Intraoperativ findet sich eine Arrosionsblutung eines Asts der A. colica media. Der ganze Bauchraum ist mit Koageln ausgefüllt. Die Ursache der Blutung ist am ehesten eine Insuffizienz der Pankreatikojejunostomie, die über eine Oberbauchperitonitis das Gefäß angedaut hat, aber das lässt sich erst auflösen, nachdem alle Koagel ausgeräumt und die Blutung umstochen worden ist. Das Querkolon kann erhalten bleiben, aber die Pankreasanastomose lässt sich unter den Schockbedingungen nicht sinnvol erneuern. Der Patient wird stattdessen pankreatektomiert.

Der weitere Verlauf wird durch eine längere Beatmung wegen einer Schocklunge und einer erschwerten Insulin- und Kaliumsubstitution als Folge der Pankreatektomie zusätzlich erschwert, doch der Mann überlebt.
Die unbefriedigende Erfolgsrate hat zu vielfachen Bemühungen zur Verbesserung der OP-Technik geführt. Im Vergleich verschiedener Rekonstruktionen nach partieller Duodenopankreatektomie konnte bislang jedoch keine Technik die Komplikationsraten entscheidend senken und sich damit als überlegen erweisen. Die Pankreatikojejunostomie als End-zu-Seit-Anastomose und die Pankreatikogastrostomie stellen derzeit die weltweit am häufigsten durchgeführten Rekonstruktionsvarianten dar. In zwei randomisierten, kontrollierten Studien lag die Komplikationsrate bei der Pankreatikogastrostomie niedriger. Zentren, welche die Pankreatikojejunostomie bevorzugen, publizieren andere Ergebnisse. Allgemein wird empfohlen, diejenige Technik zu benutzen, mit der der Operateur die größte Erfahrung besitzt.

\section{Überblick}

\section{Mögliche Folgen von Pankreasfisteln}

- intraabdomineller Abszess

- Gastroparese und Reflux

- Arrosionsblutung

- Sepsis

- intestinale Fistel 


\section{Perioperative Medizin}

Tabelle 4

Klinische Einteilung von Pankreasfisteln nach ISGPF

(International Study Group of Pancreatic Fistula).

\begin{tabular}{|c|c|c|c|}
\hline Grad & A & B & C \\
\hline klinischer Allgemeinzustand & gut & meist gut & krank/schlecht \\
\hline Spezielle Behandlung* & nein & ja/nein & ja \\
\hline US/CT (falls durchgeführt) & negativ & negativ/positiv & positiv \\
\hline $\begin{array}{l}\text { verbleibende Drainage } \\
\text { (nach } 3 \text { Wochen) }^{* *}\end{array}$ & nein & meist ja & ja \\
\hline Reoperation & nein & nein & ja \\
\hline Tod in Beziehung zu POPF & nein & nein & $\begin{array}{l}\text { möglicherweise } \\
\text { ja }\end{array}$ \\
\hline Zeichen einer Infektion & nein & ja & ja \\
\hline Sepsis & nein & nein & ja \\
\hline Wiederaufnahme & nein & ja/nein & ja/nein \\
\hline \multicolumn{4}{|c|}{$\begin{array}{l}\text { US: Ultraschall; CT: Computertomografie; POPF: postoperative Pankreasfistel } \\
\text { * teilweise (periphere) oder totale parenterale Ernährung, Antibiose, enterale Ernährung, } \\
\text { Somatostatin-Analoga und/oder minimalinvasive Drainage } \\
{ }^{* *} \text { mit oder ohne Drainage in situ }\end{array}$} \\
\hline
\end{tabular}

Die erste Folge einer Insuffizienz an der Pankreasnaht ist eine Fistel. Die Fistelrate nach Pankreaseingriff hängt von der Lokalisation der Resektion und der Definition des Begriffs Fistel ab. Neuere Übersichtsarbeiten geben eine Rate von ca. $22 \%$ an. Die heterogene Definition einer Pankreasfistel hat lange die Vergleichbarkeit von Studien erschwert. Seit 2005 existiert deshalb eine internationale Definition und Gradeinteilung. Als Fistel betrachtet man danach einen messbaren Flüssigkeitsaustritt an Tag 3 oder später, dessen Amylase-Gehalt gegenüber dem Serumwert mindestens dreifach erhöht ist. Die Schweregrade $\mathrm{A}, \mathrm{B}$ und $\mathrm{C}$ richten sich nach der klinischen Wertigkeit der Fistel aus (Tab. 4).

Wichtigste Maßnahme zur Ausheilung einer Pankreasfistel ist die Drainage des austretenden Sekrets, die häufig interventionell erreicht werden kann. Die unerkannte Fistel, die sich nicht nach außen entleeren kann, führt ansonsten zu Sekundärkomplikationen. Dazu gehören intraabdominelle Abszesse, eine Gastroparese oder auch die Arrosionsblutungen (s. Infobox „Überblick“). Arrosionsblutungen aus viszeralen Gefäßen, die sich entweder nach endoluminal oder in die freie Bauchhöhle entleeren, stellen eine akut lebensbedrohliche Situation für die Patienten dar.
Blutungskomplikationen treten nur in 6-9\% der Fälle nach pankreaschirurgischen Eingriffen auf, sind dann aber wegen des akuten Verlaufs mit einer hohen Mortalitätsrate von bis zu $50 \%$ vergesellschaftet.

Drainagen begründen sich im Rahmen von Primäreingriffen technisch in der Ableitung von Sekreten und der Anzeige von Nachblutungen oder Heilungsproblemen. Diese operationstheoretische Annahme folgt aber einer tradierten Vorstellung als einer bestätigten Evidenz. Daten, welche die Effizienz von intraabdominellen Drainagen bestätigen, existieren nicht.

- Cave. Auch die Einlage multipler Drainagen ist weder prophylaktisch noch therapeutisch in der Lage, den gesamten Bauchraum zu drainieren!

Stattdessen häufen sich Studien, die nachteilige Effekte beschreiben. Das von der dänischen Arbeitsgruppe um Kehlet inaugurierte Fast-Track-Konzept rät vom Drainageneinsatz konsequenterweise ab. Allein die engere Betrachtung der Kompartimente im Abdomen, die nicht nur der menschlichen Anatomie, sondern auch einfachen mechanischen Verschiebungen durch postoperative Bewegungen oder selbst Atemexkursionen folgt, kann diese Empfehlung grundsätzlich unterstützen. In der Pankreaschirurgie hat sich der Verzicht auf Drainagen allerdings auch international nicht durchgesetzt. Auch wenn hier der endgültige Nachweis von Vorteilen aussteht, gibt es Studien, die aus ihren Ergebnissen das Gegenteil ableiten. Vielfach wird in der Pankreaschirurgie empfohlen, Drainagen zu legen, aber nach Möglichkeit wieder früh zu entfernen. Viel zu oft leiten Drains gar nicht das ab, was sie ableiten sollen. Das führte auch in unserem Fall zeitweilig zu einer falschen Sicherheit. Der Einsatz von Drainagen erfordert in allen Fällen eine strenge Abwägung von Risiko und Nutzen.

Überblick

\section{Mögliche Komplikationen von Drainagen}

- Drucknekrosen

- Darmfisteln

- Gefäßarrosionen

- aszendierende Infektionen

- Hernien

- Schmerzen

- Drainageretraktion, Fremdkörperverbleib

- Berichtswesen - mehr Komplikationen mit Unerfahrenen? 
Ein entscheidendes Kriterium für die Erfolgsrate in der Erfassung und Behandlung von Komplikationen scheint die Informationskette zu spielen. „Wen informiere ich wann und wie?“ ist hier die Kernfrage. Patienten werden heute im Rahmen ihres Krankenhausaufenthalts mehrheitlich diskontinuierlich behandelt: Der Patient leidet, heilt und genest 24 Stunden pro Tag und zwar am Stück der Arzt behandelt je nach Modell und vertraglicher Arbeitszeit 6, 8 oder 12 Stunden davon.

Die räumliche und zeitliche Trennung von Patient und Arzt ist in vielerlei Hinsicht sinnvoll, und das nicht nur für das Zeitbedürfnis biologischer Heilvorgänge. Auch die Kräfte der Ärzte müssen geschont werden, um sich erholen zu können, selbst wenn die idealen Zeitfenster für Arbeitsalltag, Dienst und Erholung international sehr kontrovers diskutiert werden (die europäischen Arbeitsmodelle werden in den USA als nicht praktikabel beurteilt). Die fehlende Parallelität der Ereignisse - die Krankheit hält sich nicht an die tariflichen Arbeitszeiten - stellt einen besonders hohen Anspruch an die Informationssysteme und das Meldewesen. Die allseitige Verfügbarkeit mobiler Telefon- und Internetverbindungen sowie computergestützter Datenbanken kann dabei maximal ein Hilfsmittel sein, stellt aber keine echte Sicherung dar. Dazu kann ein ständiges Informieren den Informierten auch ermüden. Irgendwann hört man nicht mehr richtig zu. Also ist wann wichtiger als kann.

Das erfolgreiche Komplikationsmanagement hängt auch davon ab, ab wann der Erstbehandler seine Grenze erkennt und den Erfahrenen zu Hilfe ruft. Der direkte Zusammenhang zwischen ärztlicher Erfahrung und Komplikationsrate wird allerdings ebenfalls kontrovers diskutiert. Zumindest unter professioneller Supervision müssen die jungen Kollegen keine höhere Rate an Zwischenfällen fürchten. Auf der anderen Seite stehen Fallzahl und Komplikationsrate für viele chirurgische Prozeduren in einem engen Kontext. Die Mindestzahldebatte

\section{Komplikationsmanagement}

\section{Strategisches Vorgehen im anspruchsvollen Notfall}

- vorurteilsfreie Befunderhebung

- Datensammlung und zielorientierte Datenergänzung

- Überprüfung der Arbeitshypothese

- bei Bestätigung: konsequente Therapie

- bei Ablehnung: Reset und Neustart, ggf. Hilfe anfordern fußt auf dieser Annahme. Die Supervision ist also der Schlüssel zum Erfolg.

Das Leben rettet in unserem Fallbeispiel der Oberarzt am Telefon, der dem Patienten den fatalen Weg durch die CT erspart und direkt die Operation anordnet.

\section{Diagnostisches und therapeutisches Vorgehen}

Die schnellste und einfachste bildliche Darstellung von abdominellen Zuständen gelingt mit der Sonografie. In der Hand des Erfahrenen stellt die Ultraschalluntersuchung ein sehr wertvolles Instrument in der Notfalldiagnostik dar. Aber wie so oft ist es der menschliche Faktor, der den Unterschied ausmacht. Die Ergebnisse sind in jedem Bereich sehr untersucherabhängig. Dazu lassen sich manche Veränderungen nur erschwert abbilden, da sie sich in der Echodichte nicht ausreichend von der Umgebung abheben (s. Box „Zielgerichtete Diagnostik").

Zielgerichtete Diagnostik

\section{Sonomorphologische Phänomene bei der Darstellung von Darm als intraabdominelle Hämatome}

- unregelmäßig begrenzte, echoarme Anteile

- unterschiedliche Mengen an Binnenreflexen

- evtl. hyperechogene Strukturen

- dorsale Schallverstärkungen

In unserem Fallbeispiel lassen sich die festen, nicht sedimentierten Hämatomanteile vom diensthabenden Chirurgen nicht von den gefüllten Darmanteilen abgrenzen. Die Untersuchung bietet statt klaren Hinweisen nur trügerische Sicherheit. In einer solchen Situation ist die Eskalation der Diagnostik mittels einer CT sicher nicht grundsätzlich falsch. Hier ließe sich das Hämatom wahrscheinlich zweifelsfrei identifizieren. Allerdings schließt der exponentiell steigende Druck des Notfalls jeden weiteren Zeitverlust aus. Der Mann muss in den OP gebracht werden.

\section{- Revisionsoperation}

Die Behandlungsstrategie von Fistel, Insuffizienz und ihren Folgen hat sich in den letzten Jahren stark gewandelt. Da in $80 \%$ der Fälle Low-Output-Fisteln vorliegen, reicht hier das Sichern eines ausreichenden Abflusses, z. B. durch eine Drainage, aus. Selbst die Nahrungskarenz ist in 


\section{Perioperative Medizin}
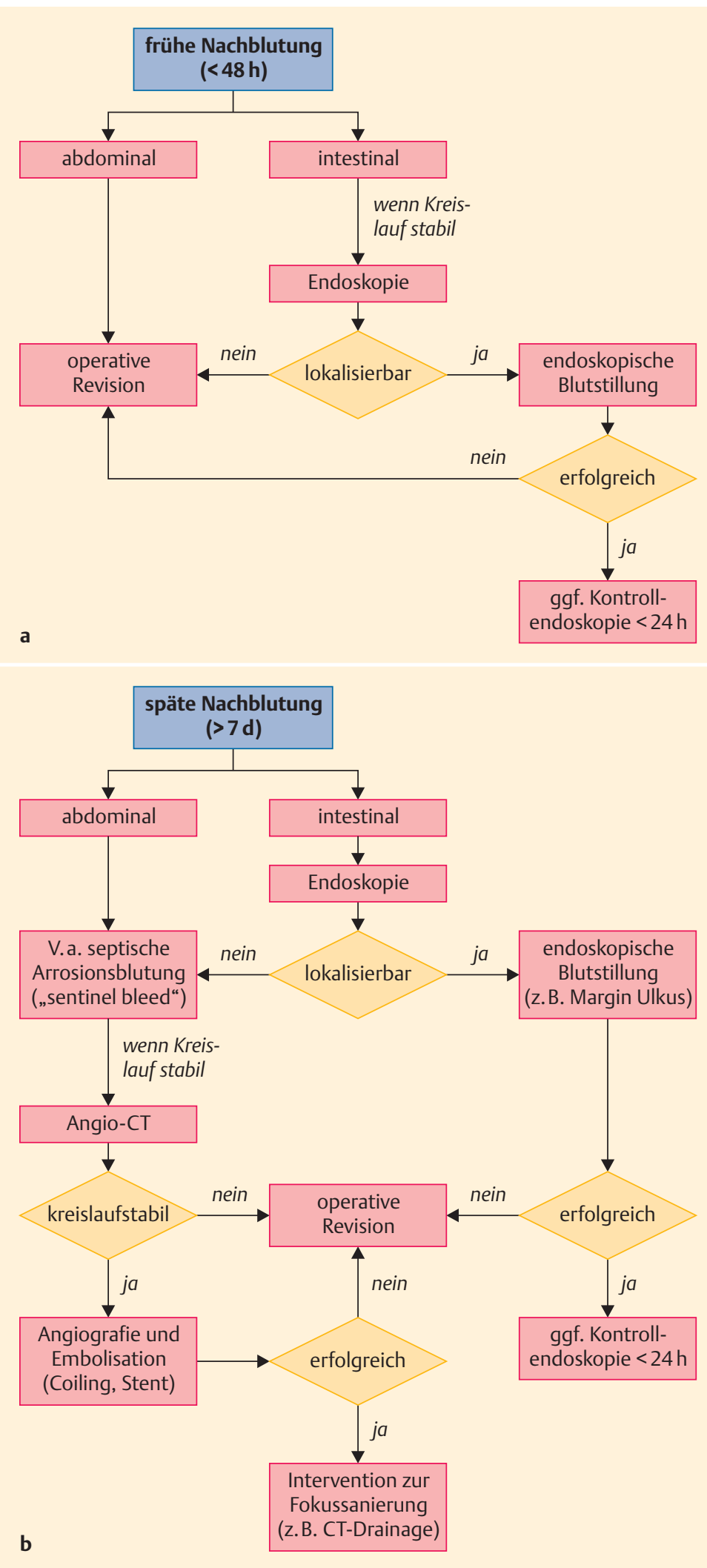

Abb. 2 - Therapeutischer Algorithmus bei früher (a) und später (b) Nachblutung nach Pankreaskopfresektion (modifiziert nach Kleespies et al. 2007).
Abhängigkeit von der Lokalisation der Fistel nicht mehr zwingend notwendig. Insbesondere CT-gesteuert einlegte Drainagen haben die Rate der Relaparotomien gesenkt.

Operative Revisionen ermöglichen grundsätzlich eine Anpassung an den individuellen Befund und Situs. Selbst komplett neue Rekonstruktionen werden in der Literatur beschrieben. Doch oft ist eine Restpankreatektomie unumgänglich, da Durchblutungsstörungen am Darm, ödematöse Gewebsveränderungen oder Nekrosen am Pankreas oder aber auch eine instabile Kreislaufsituation die Heilungsbedingungen drastisch verschlechtern. In der Sepsis ist diese Salvage-Restpankreatektomie in der Regel unumgänglich, um das Leben des Betroffenen zu retten.

Postoperative Blutungen treten akut auf und verlangen auch ein akutes Handeln. Bei stabilen Kreislaufverhältnissen ist die angiografische Intervention, z. B. über eine Embolisation oder ein Coiling, die eleganteste Methode, die Blutung zu stoppen (Abb. 2). Das technisch anspruchsvolle Verfahren limitiert sich jedoch durch die Tatsache, dass nicht jedes Gefäß folgenlos verschlossen werden kann. Das betrifft vor allem die Leberpforte, also die Aa. hepaticae, deren Verschluss bei fehlender akzessorischer Versorgung auch zu Leberteilnekrosen und -abszessen führen kann. Außerdem muss sich der Blutstillung zwingend eine Sanierung des ursächlichen, septischen Fokus anschließen. Unter weniger dramatischen Umständen als in unserem Fall hätte eine Arrosionsblutung z. B. der A. gastroduodenalis auch angiografisch gestillt werden können. Dies trifft vor allem in den Fällen $z u$, in denen z.B. nach bereits erfolgter Restpankreatektomie die Versorgung einer Insuffizienz nicht mehr erforderlich ist.

Die Mortalität einer Relaparotomie in der akuten Blutung liegt allerdings hoch. Die arrondierten Gefäße müssen in der Regel durch Umstechung verschlossen werden, was der Durchblutung ebenfalls nicht förderlich ist, weswegen viele Autoren auch hier eine komplette Restpankreatektomie empfehlen.

\section{- Folgen der Pankreatektomie}

\section{Substitution der Pankreasfunktion}

Überlebt der Patient die Notfallsituation durch eine totale Pankreatektomie, so muss er danach die anspruchsvolle Substitution der Pankreasfunktion überstehen. Hier steht natürlich die Therapie mit Insulin und Pankreasfermenten im Vordergrund, die ja bei subtotalen Pankreasinsuffizienzen ebenfalls notwendig ist. 
Die Substitution der Pankreasenzyme richtet sich nach der Nahrungszusammensetzung und kann analog zur Therapie der Mukoviszidose beim Essen errechnet und mittels Stuhlschau angepasst werden. Allerdings führt der komplette Verlust des Organs zu einem pankreopriven Diabetes mellitus (Typ 3c), dessen Einstellung besonders schwierig ist. Die Patienten neigen wegen eines parallelen Mangels von Glukagon zu Hypoglykämien. Zudem führen die als Folge der Resektionen von Duodenum, Gallenblase und -gang und eventuell Teilen des Magens veränderten Verdauungs- und Resorptionsabläufe zu einer weiteren Labilität des Glukosespiegels. Die meisten Patienten müssen wegen dieser komplexen Therapie dauerhaft in enger ärztlicher Betreuung bleiben.

\section{Splenektomie}

Die Milz wird bei einer Pankreatektomie in der Regel auch entfernt. Nur wenige Operateure beschreiben den Verbleib der Milz, die dann aber nur noch über die Aa. gastricae breves ernährt wird.

Die Splenektomie kann zum Postsplenektomie- oder auch OPSI-Syndrom („overwhelming post-splenectomy infection“) führen. Die immer septisch verlaufende Infek- tion ist lebensbedrohlich. Zur Prophylaxe werden die Patienten gegen Meningokokken, Pneumokokken und Haemophilus influenzae geimpft. Die Impfung ist erst dann sinnvoll, wenn die Patienten immunologisch wieder in der Lage sind, Antikörper zu bilden. Dazu muss bei bakteriellen Infekten lebenslang eine frühzeitige Antibiotikatherapie eingeleitet werden.

Post Splenektomie: trivalente Impfung nicht vergessen!

\section{Schmerzen nach Gastrektomie: Schmerztherapie}

\section{Hintergrund}

Der professionelle Umgang mit Schmerzen lindert nicht nur Leiden, sondern führt auch zu einer verbesserten Heilung. Viele Eingriffe in der Tumorchirurgie sind ohne eine perfektionierte Schmerztherapie gar nicht durchführbar, da die systemischen Belastungen nicht nur bei 2-Höhlen-Eingriffen sonst zu hoch wären. Es gibt sogar eine Kampagne für das „schmerzfreie Krankenhaus“.

Fallbeispiel 7

Die Dienstärztin wird am 4. postoperativen Tag nach Gastrektomie am Sonntagvormittag zu einer 56-jährigen Patientin auf eine periphere Station gerufen. Die Frau klagt über Schmerzen im Oberbauch, die selbst das Atmen behindern. Das Pflegepersonal hat die Patientin an den Waschtisch mobilisiert, was nur mit großer Mühe gelang. Der Verlauf war ansonsten bisher unkompliziert - die Frau darf eigentlich seit heute schluckweise trinken, nimmt wegen der Schmerzen aber nichts zu sich.

Die Ärztin orientiert sich über die Vitalparameter, erfragt den Schmerzscore (6) und ordnet dann schon am Telefon eine Infusion mit $2 \mathrm{~g}$ Metamizol an. Bei ihrer Ankunft hat sich die Situation kaum verbessert. Die Patientin klagt immer noch über Oberbauchschmerzen, die sie bei der Bewegung und der Atmung behindern. Besonders schmerzt die Austrittsstelle der Drainage. Der Blutdruck liegt bei $140 / 90 \mathrm{mmHg}$, die
Frau ist etwas tachykard. Die Sauerstoffsättigung hat die betreuende Krankenpflegerin mit 2 I Sauerstoff über die Nasenbrille auf $94 \%$ gehoben.

Der Bauch ist druckempfindlich, aber weich und zeigt keinen Peritonismus. Die Wunde ist reizlos, und die Drainage fördert serös-blutige Flüssigkeit. Die Patientin hat kein Fieber, und das Labor vom Vortag war weitgehend unauffällig. Es liegt ein PDK, der auch alarmfrei läuft. Die Lunge auskultiert sich in den oberen Abschnitten unauffällig. Die Unterfelder sind aber nicht sicher zu beurteilen - die Patientin atmet nicht tief genug durch.

Die Chirurgin bleibt aufmerksam und sucht weiter. Nach intraoperativer ZVK-Anlage ist ein Röntgenbild des Thorax durchgeführt worden, das keine Auffälligkeiten zeigt. Der einliegende Urinkatheter fördert klaren Urin.

Anamnestisch und laut Aktenlage sind außer einer Hypertonie keine relevanten Vorerkran- kungen bekannt. Die Patienten hat eine antihypertensive Dauermedikation, die derzeit aber pausiert. Die Kollegin wirkt jetzt beruhigend auf die Frau ein und erklärt, bisher nichts Besorgniserregendes gefunden zu haben, dass sie ihre Beschwerden aber dennoch ernst nimmt.

Sie entschließt sich, den Akutschmerzdienst zur Überprüfung des PDK hinzuzuziehen. In der Zwischenzeit infiltriert sie die Austrittsstelle der Drainage subkutan mit einem Lokalanästhetikum. Der zügig eintreffende anästhesiologische Kollege vom Schmerzdienst erhöht die Laufrate des PDK und titriert die Piritramid-Dosis bettseitig unter Monitorüberwachung, bis die Patientin schließlich deutlich schmerzgelindert ist. Danach lassen sich auch die unteren Lungenabschnitte unauffällig auskultieren. Am späten Abend besucht die Dienstärztin die Station erneut und findet eine deutlich beschwerdegebesserte Patientin vor. 
Das ist vielleicht illusorisch, aber sicher sollte man sich bemühen, jede Behandlung oder Operation mit minimalen Beeinträchtigungen ablaufen zu lassen.

Viele Studien bestätigen in diesem Kontext, dass postoperative Schmerzen mehr Komplikationen und eine verzögerte Wundheilung auslösen. Dazu sinken natürlich Patientenkomfort und -zufriedenheit, und schließlich verlängert sich der Krankenhausaufenthalt. Eine angemessene Schmerztherapie zu versäumen ist also nicht nur inhuman, sondern auch medizinisch kontraproduktiv. Zudem sind die Möglichkeiten der Schmerzprophylaxe und -therapie heute sehr breit gefächert und auch in der Kombination eher simpel.

Eine strukturierte Schmerztherapie ist integraler Bestandteil der viszeralchirurgischen Behandlung.

\section{Diagnostisches Vorgehen}

\section{Was ist Schmerz, und wie wird er gemessen?}

Um akute Situationen zu lösen - und genau darum geht es im Notfall - ist es wichtig zu akzeptieren, dass Schmerzempfinden individuell ausprägt ist. Es wird durch eine emotionale Bewertung, frühere Schmerzerfahrungen und die situative Einordnung moduliert. Die Empfindung ist ganz wesentlich von der individuellen Situation und den Umständen abhängig. Jeder Mensch erlebt und wertet Schmerz anders. Hat ein chronisch kranker Patient zu Beginn seiner Karriere kritische Situationen und Eingriffe mit stabiler Selbstdisziplin gemeistert, kann sich diese Ruhe über die Jahre aufbrauchen

\section{a visuelle Analogskala (VAS)}

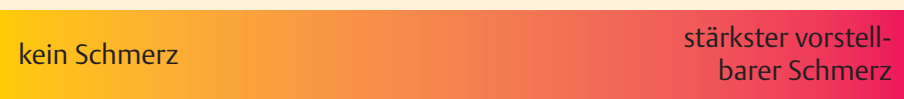

b numerische Rating-Skala (NRS)

\begin{tabular}{|l|l|l|l|l|l|l|l|l|l|l|}
\hline 1 & 2 & 3 & 4 & 5 & 6 & 7 & 8 & 9 & 10 \\
\hline kein Schmerz & & & & & & & \\
\hline
\end{tabular}

c verbale Rating-Skala (VRS)

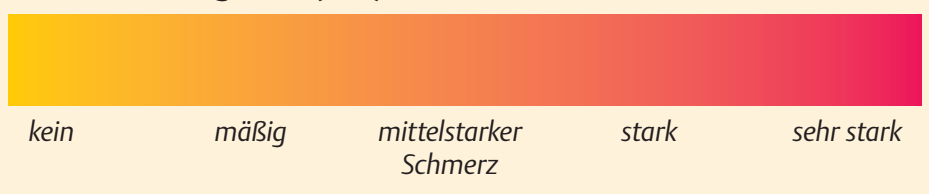

Abb. 3 - Skalen zur Messung der Intensität postoperativer Schmerzen: a Numerische RatingSkala (NRD). b Visuelle Analogskala (VAS). c Verbale Rating-Skala (VRS). und eine übergroße Empfindlichkeit entstehen. Im Schock kann dagegen jedes Schmerzempfinden nahezu ausgeschaltet sein.

Jedes Individuum besitzt eine individuelle Leidensfähigkeit. Jede Schmerzangabe muss deswegen ernst genommen und abgeklärt werden. Dies gilt natürlich insbesondere für Patienten nach operativen Eingriffen. Inadäquate oder mit den Standardmitteln nicht beherrschbare Schmerzen nach Operationen fordern immer den Ausschluss aller möglichen Komplikationen. In unserem Beispiel schließt die Dienstärztin strukturiert und konsequent eine solche postoperative Komplikation aus.

Jede Schmerzäußerung muss ernst genommen und
abgeklärt werden!

Der subjektiv empfundene Schmerz fordert zur strukturierten Einschätzung eine Messung. Es hat sich bewährt, die Intensität in Form einer numerischen, visuellen oder verbalen Rating-Skala zu messen. Der Schmerzcharakter kann zudem in freien Begriffen ergänzt werden. Bei Kindern wendet man eine eher altersgerechte Schmerzmessung mit der „Smiley-Analogskala“ an.

In allen Altersstufen unterstützen die Messungen von Vigilanz, Atmung, Herzfrequenz und Blutdruck die objektivierte Einschätzung. Die reine Fremdeinschätzung durch das medizinische Personal ist dagegen nicht ausreichend, denn auch hier verfälschen persönliche Erfahrungen und Lebenseinstellungen die Realität.

\section{- Schmerzskalen}

Die anhand einer Rating-Skala (Abb. 3) eingeschätzten Schmerzwerte sollten in der Krankenakte dokumentiert werden. Die Effekte sind nachhaltig, denn alle profitieren: Die Patienten fühlen sich ernst genommen - man glaubt ihnen und ist bemüht, Hilfe zu leisten. Und die Therapeuten erhalten einen Vergleichswert, der es ihnen erlaubt, die Verhältnismäßigkeit der Mittel einzuhalten.

\section{Therapeutisches Vorgehen}

\section{- Perioperative Schmerzvermeidung}

Schmerzen kann man auch als Chirurg auf verschiedene Arten vermeiden. Primär ist eine Begrenzung des Zugangstraumas anzustreben. Die minimalinvasive Chirurgie reduziert in vielen Studien den postoperativen Schmerzmittelverbrauch deutlich, obwohl der Eingriff an den inneren Organen der konventionellen Chirurgie entspricht. Aber auch in offenen Verfahren kann man durch 
die Schnittführung postoperative Schmerzen senken. Vereinfacht schneidet jeder quere Zugang besser als ein medianer Schnitt ab - pararektale und transrektale Schnitte sind auch aus anderen Gründen obsolet.

Die prä- oder postoperative Injektion von Lokalanästhetika im Operationsbereich hat ebenfalls einen positiven Effekt auf das postoperative Schmerzniveau. Die naheliegende Wirkung wird unterschätzt, obwohl der Aufwand gering ist.

Eine erhebliche Erleichterung bietet der Einsatz eines Periduralkatheters (PDK). Die kontinuierliche Applikation von Lokalanästhetika in den Periduralraum schaltet Schmerzafferenzen auf Rückenmarkniveau aus. Der Organismus reagiert dann auf den Eingriff nicht mit einem erhöhten Sympathikotonus, der alle intestinalen Funktionen zugunsten der lebenswichtigen Organerhalte dämpft. Bei der thorakalen Anwendung steht die die abdominelle Sympathikolyse im Vordergrund. Die intestinalen Organe arbeiten als Folge dieser Sympathikolyse wie gewohnt weiter. Insbesondere der Magen-DarmTrakt transportiert, verdaut und scheidet weiter aus, was für die Heilung und Erholung von zentraler Bedeutung ist. Das Verfahren reduziert dadurch auch den Verbrauch sonstiger Schmerzmittel. Der Einsatz eines Periduralkatheters ist zentraler Teil der „Fast-Track-Rehabilitation“.

Ein lumbal positionierter PDK ist in der Viszeralchirurgie dagegen weniger gebräuchlich, da er nur eine geringe Sympathikolyse erzeugt, stattdessen aber die Blasenentleerung und Beinmotorik behindert. Zur Ergänzung der PDK-Analgesie können Morphine zur Anwendung kommen. Falls die peridurale Analgesie über eine Kombination von Lokalanästhetikum und Opioid gesteuert wird, bevorzugt man wie in unserem Fall eher Metamizol, da dann die Opioidrezeptoren durch die systemische Resorption im Periduralraum zumindest anteilig schon besetzt sind.

Nach kleineren Eingriffen oder Traumata wird die lokale Kälteanwendung als schmerzlindernd empfunden. Die Wirkung setzt schnell ein, hält aber nur begrenzt an.

Die Physiotherapie besitzt in der perioperativen Therapie je nach Eingriff einen variablen Stellenwert und nutzt verschiedene Wirkprinzipien. In der Viszeralchirurgie mindern alle Maßnahmen Fehlhaltungen, Verspannungen und Immobilität und lindern so schmerzinduzierende Zustände.

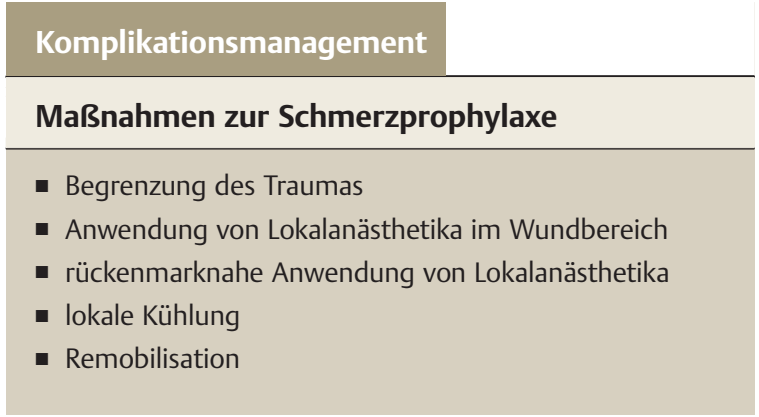

Bei allen Einsätzen in der Schmerzbehandlung sollte man die suggestive Kraft des Arztes nicht außen vor lassen. Der „weiße Kittel“ verleiht dank Image und Nimbus viel Energie, die man wie ein Placebo sehr positiv einsetzen kann. Die Wirkmechanismen sind Jahrtausende bekannt, werden heute aber oft aus technokratischen Gründen vernachlässigt. Zur Suggestion gehört auch, dass die Befunderhebung und die Schmerztherapie in engem zeitlichem Zusammenhang bleiben.

Das Gegenstück sind sogenannte Nocebos: „Vielleicht hilft Ihnen diese Tablette jetzt mal“ ist dem euphorischen „damit geht es Ihnen gleich viel besser“ deutlich unterlegen (Tab. 5).

Tabelle 5

\section{Nocebos in der Schmerztherapie.}

\begin{tabular}{|c|c|}
\hline Aussage & möglicher Effekt \\
\hline „Vielleicht hilft dieses Mittel?“ & Verunsicherung \\
\hline
\end{tabular}

„Versuchen Sie, die Schmerzmittel regelmäßig zu nehmen.“

"Ich hole noch was aus dem Giftschrank.“ doppeldeutig

"Jetzt schläfern wir Sie ein, gleich ist alles vorbei.“

„Das tut schon immer höllisch weh.“ negative Suggestion

„Das tut gar nicht weh.“ unwirksame Verneinung

„Das kann man aber aushalten.“

„Sie sind ein Risikopatient.“ 


\section{Perioperative Medizin}

\section{Welches Schmerzmedikament wann und wie?}

Die Wahl des Analgetikums richtet sich nach der zu erwartenden Schmerzstärke. Ein weiteres Kriterium ist die Applikationsform, und schließlich geht es auch um die Schnelligkeit des Wirkeintritts. Im Notdienst sollte man eine gesicherte Resorption oder sonstige Aufnahme und einen zügigen Wirkeintritt bevorzugen.

Die Zeit ist meistens knapp, und die Patienten sind oft gestresst und ängstlich. Alle Maßnahmen werden von einer kurzen Erklärung begleitet. Dabei geht es im Wesentlichen darum, Sicherheit zu erzeugen und im Notdienst Vertrauen zu schaffen. Komplizierte Einstellungen verlangen auch deshalb eine Beschreibung, damit eine ausreichende Compliance erreicht wird.

Als Basismedikation bei viszeralen Schmerzen bietet sich als peripheres Analgetikum Metamizol.

Metamizol ist dabei aber ein „typisch deutsches“ Medikament. In Skandinavien, den USA und vielen anderen Ländern hat die mögliche Nebenwirkung der Agranulo-

\section{Prinzipien}

\section{Arzneimittelkombination}

In der kombinierten Schmerztherapie nutzt man synergetische Effekte. Die früher eher verpönte Kombination unterschiedlicher Präparate gehört heute zum Standard. Dadurch ergänzen sich unterschiedliche Wirkprinzipien, ohne dass die Wirkstoffe um den gleichen Rezeptor oder Enzymweg konkurrieren. In der klassischen Form findet man im Krankenhaus die parallele Gabe von Morphinen, peripher wirksamen Analgetika und Entzündungshemmern. zytose zu einer Zulassungssperre geführt. Die fachliche Beurteilung hat in Deutschland zu einer anderen Einschätzung geführt, aber Patienten aus Herkunftsländern mit Zulassungssperre sollten eher nicht mit Metamizol behandelt werden. Alternativ steht Paracetamol in der gleichen Dosierung zur Verfügung. Eine gute Kombinationsmöglichkeit bieten Antiphlogistika wie z.B. Ibuprofen oder Diclofenac.

Die häufig automatisch eingesetzte Komedikation zur Ulkusprophylaxe wird bei kurzzeitiger Anwendung heute kritischer gesehen. In der nächsten Stufe kommen zusätzlich Opioide zum Einsatz. Opioide lassen sich am besten steuern, die Präparate werden dazu auch nach ihrem analgetischen Äquivalentwert im Verhältnis zu Morphin eingeteilt. Abb. 4 zeigt das Stufenschema zur Therapie chronischer Schmerzen nach WHO, die Adjuvanzien in der Schmerztherapie sind in der Infobox dargestellt. Bei akut einsetzenden, starken Schmerzen kann die Eskalation der Mittel dagegen oft nicht eingehalten werden.

\section{Überblick}

\begin{tabular}{l} 
Adjuvanzien in der Schmerztherapie \\
\hline - Laxanzien \\
- Antiemetika \\
- Magenschutz \\
- Koanalgetika \\
- Antidepressiva \\
- Lokalanästhetika (LA) \\
- Antikonvulsiva \\
- Kortikosteroide \\
- Spasmolytika \\
- Bisphosphonate
\end{tabular}

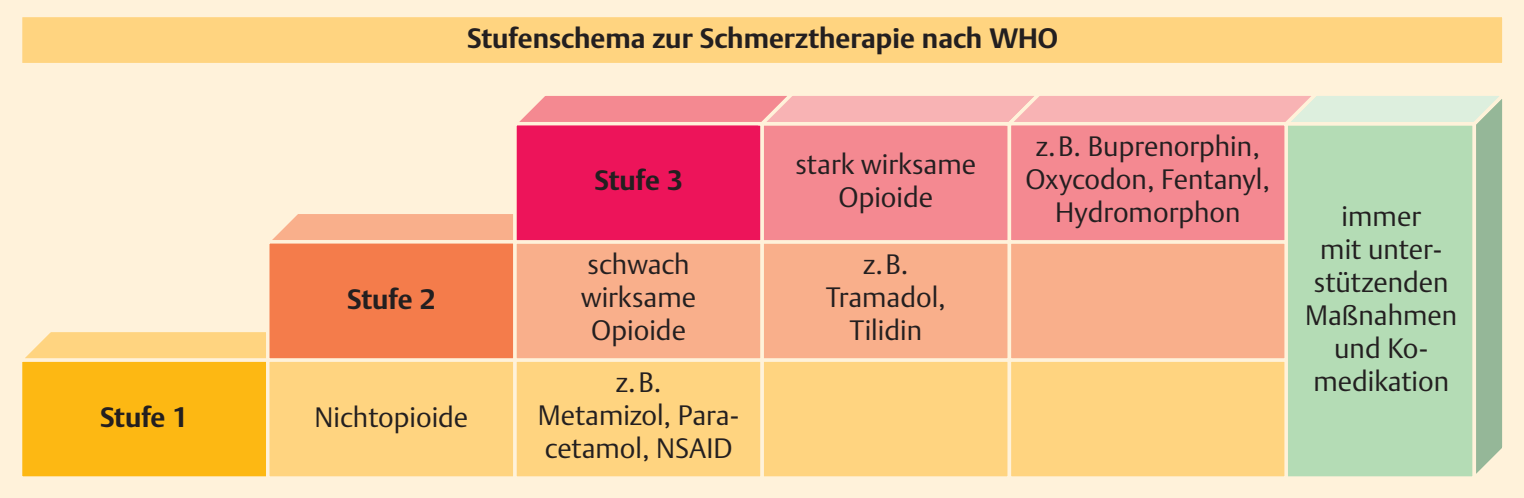

Abb. 4 - Stufenschema zur Therapie chronischer Schmerzen nach WHO (immer mit unterstützenden Maßnahmen und Komedikation). 
Hier nutzt man primär die Wirkung des Opioids, die man danach mit Nichtopioiden ergänzen kann.

Alle Analgetika verlangen auch im Notdienst vor dem Einsatz einen Ausschluss von Allergien.

Die typischen Nebenwirkungen lassen sich bei ausreichender Sachkenntnis sofort mit behandeln.

Patienten mit Periduralkatheter (PDK), patientenkontrollierter epiduraler Analgesie (PCEA) und Regionalanästhesiekathetern (RAK) werden in den meisten Kliniken von der Anästhesie betreut. Diese Aufteilung kann bei Funktionsproblemen wie in unserem Fall zu Versorgungslücken führen. Die diensthabende Ärztin überbrückt hier richtig mit einer Metamizol-Infusion.

Abdominalschmerzen werden oft immer noch - aus Angst, wichtige Befunde zu maskieren - nur zögerlich analgetisch therapiert. Diese Haltung ist wissenschaftlich nicht begründbar und damit medizinisch falsch.

Die Gabe eines Analgetikums gehört in der Akutsituation immer zu den ersten ärztlichen Aufgaben.

Interessenskonflikt: Die Autoren bestätigen, dass kein Interessenskonflikt vorliegt.

\section{Korrespondenzadresse}

Dr. Carsten J. Krones

Klinik für Allgemein- und Viszeralchirurgie,

Katholische Stiftung Marienhospital Aachen

Zeise 4

52066 Aachen

Telefon: 049-241/6006-1200

Fax: $\quad 049-241 / 6006-1209$

E-Mail: Carsten.Krones@marienhospital.de

\section{Zum Weiterlesen und Vertiefen}

Ahmed N, Devitt KS, Keshet I. A systematic review of the effects of resident duty hour restrictions in surgery: impact on resident wellness, training, and patient outcomes. Ann Surg 2014; 259: 1041 - 1053

Arbeitsgemeinschaft Medizinisch-Wissenschaftlicher Fachgesellschaften - AWMF. S3-Leitlinie Behandlung akuter perioperativer und posttraumatischer Schmerzen. AWMF-Register Nr. 041/001

Attard AR, Corlett MJ, Kidner NJ et al. Safety of early pain relief for acute abdominal pain. BMJ 1991; 305: 554-556

Bassi C, Dervenis C, Butturini G et al. Postoperative pancreatic fistula: an international study group (ISGPF) definition. Surgery 2005; 138 : $8-13$

De Santibanes M, Alvarez FA, Sieling E et al. Postoperative complications at a university hospital: is there a difference between patients operated by supervised residents vs. trained surgeons? Langenbecks Arch Surg 2015; 400: $77-82$

Diener MK, Tadjalli-Mehr K, Wente MN et al. Risk-benefit assessment of closed intra-abdominal drains after pancreatic surgery: a systematic review and meta-analysis assessing the current state of evidence. Langenbecks Arch Surg 2011; 396: 41 - 52

Gómez T, Palomares A, Serradilla M et al. Reconstruction after pancreatoduodenectomy: Pancreatojejunostomy vs pancreatogastrostomy. World J Gastrointest Oncol 2014; 6: 369-376

Graat LJ, Bosma E, Roukema JA et al. Appendectomy by residents is safe and not associated with a higher incidence of complications: a retrospective cohort study. Ann Surg 2012; 255: 715 - 719

Harnoss JC, Ulrich AB, Harnoss JM et al. Use and results of consensus definitions in pancreatic surgery. Surgery 2014; 155: 47-57

Kleespies A, Eichhorn M, Seeliger $\mathrm{H}$ et al. Klinische Anastomosenprobleme in der Pankreaschirurgie. Viszeralchirurgie 2007; 42: 1-11

Krones C], Klink CD, Lambertz A. Management häufiger postoperativer Probleme und Komplikationen - Teil 1. Allg Viszeralchir up2date 2015; 9: 19-38

Malleo G, Pulvirenti A, Marchegiani G et al. Diagnosis and management of postoperative pancreatic fistula. Langenbecks Arch Surg 2014; 399: $801-810$

McMillan MT, Vollmer CM. Predictive factors for pancreatic fistula following pancreatectomy. Langenbecks Arch Surg 2014; 399: $811-824$

Rajarm R, Chung JW, Jones AT et al. Association of the 2011 ACGME resident duty hour reform with general surgery patient outcomes and with resident examination performance. JAMA 2014; 312: 2374-2384

Rüsch D, Eberhart LH, Wallenborn J et al. Nausea and vomiting after surgery under general anesthesia: an evidence-based review concerning risk assessment, prevention, and treatment. Dtsch Ärztebl Int 2010; 107: $733-741$

Schwenk M, Schinkel B. Perioperative Schmerztherapie. Chirurg 2011; 82: $539-556$

Wehner S, Vilz TO, Stoffels B et al. Immune mediators of postoperative ileus. Langenbecks Arch Surg 2012; 397: 591 - 601 


\section{Perioperative Medizin}

\section{CME-Fragen}

\section{CME.thieme.de}

\section{CME-Teilnahme}

- Viel Erfolg bei Ihrer CME-Teilnahme unter http://cme.thieme.de

- Bitte informieren Sie sich vorab online über die Gültigkeitsdauer.

- Sollten Sie Fragen zur Online-Teilnahme haben, unter http://cme.thieme.de/hilfe finden Sie eine ausführliche Anleitung.

Wie lange ist die peristaltische

Bewegung des Magen-Darm-

Traktes nach größeren operativen

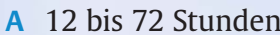

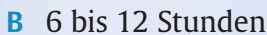

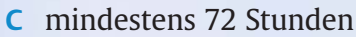

Eingriffen herabgesetzt?

D maximal 12 Stunden

E 24 bis 48 Stunden

Was ist keine Ursache von postoperativer Übelkeit und Erbrechen?
A Magen-Darm-Atonie
B Blutzuckerentgleisungen
C postoperatives Fieber
D Postoperative Nausea and Vomiting (PONV)
E Hypotonie

\section{Warum sollen Morphine bei} der Symptomlinderung der Atonie und Übelkeit zunächst nicht bevorzugt werden?
A Weil sie die Übelkeit verstärken können.

B Weil sie sehr teuer sind.

C Weil die Nebenwirkungen bei allen Wirkstoffen gleich sind.

D Weil sie die Paralyse verstärken können.

E Weil es nur einen Wirkstoff gibt.

Eines ist kein Risikofaktor für eine postoperative Pankreasfistel. Welches?

A niedriges Serumalbumin

B weibliches Geschlecht

C schmaler Pankreasgang

D Linksresektion

E BMI und Stärke der perirenalen Fettschicht

Wie hoch geben neuere Übersichtsarbeiten die Fistelrate nach Pankreaseingriffen an?

A ca. $0,2 \%$

B ca. $5 \%$

C ca. $22 \%$

D ca. $2 \%$

E ca. $52 \%$ 
Was ist keine typische Folge einer Pankreasfistel?
A Arrosionsblutung
B intestinale Fistel
C Sepsis
D intraabdomineller Abszess
E Übelkeit und Erbrechen

Wie häufig treten nach pankreaschirurgischen Eingriffen Blutungskomplikationen auf?
A $<1 \%$ der Fälle
B in bis zu 1-2\% der Fälle
C in bis zu 6-9\% der Fälle
D in bis zu 6\% der Fälle
E in bis zu 7-10\% der Fälle

Eine der folgenden Maßnahme ist nicht geeignet, um Schmerzen durch chirurgische Eingriffe zu vermindern. Welche?

M.
A minimalinvasive Eingriffe
B lokale Kälteanwendung
C Einsatz eines Periduralkatheters
D mediane Schnittführung
E prä- oder postoperative Injektion von Lokalanästhetika

\section{Welcher Wirkstoff bietet sich bei viszeralen Schmerzen für die erste Basismedikation an?

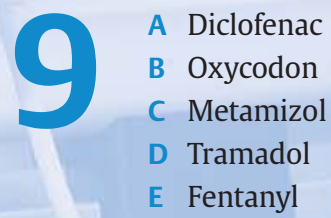

Einer der folgenden Faktoren ist keine Folge postoperativer Schmerzen. Welcher?

A niedrige Patientenzufriedenheit

B vermehrte Komplikationen

C verlängerter Krankenhausaufenthalt

D verzögerte Wundheilung

E höherer Patientenkomfort 